\title{
Application Research on Monitoring Cloud Platform of Bridge Construction Based on BIM
}

\author{
ZHI Peng ${ }^{1, a}$, SHI Tianyun ${ }^{2, b}$, WANG Wanqii, c, WANG Huilin ${ }^{4, d}$ \\ ${ }_{1,2,3,4}$ China Academy of Railway Sciences, Beijing 100081;

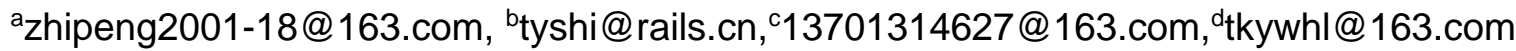

\begin{abstract}
Keywords: Bridge construction; BIM; Cloud platform; Internet of things; B/S Supervision platform Abstract. Based on BIM, cloud platform, internet of things and other advanced technology, this study establishes a monitoring cloud platform used in the bridge construction. It solved the key technology application issues such as formulating bridge BIM implementation standards and engineering breakdown structure coding, Quickly bridge modeling based on Tekla, bridge model collision check and lightweight treated, implementing visualization of bridge construction progress control, Internet of things about bridge health monitoring implementing visualization of bridge construction progress control, Internet of things about bridge health monitoring instrumentation and closing loop of safety $\&$ quality issue. In practice, the platform collects flexible and accurate information of bridge construction in time, realizes the data sharing of information, improves the collaborative operation efficiency of cooperated-building parties. Also, increases control effectively about difficult point of bridge risk source, the key progress of the task and the problems of safety and quality too.
\end{abstract}

\section{Introduction}

In recent decades, a series of long-span Road-Railway bridges has been constructed, such as Nanjing Dashengguan Yangzi River Bridge, Shanghai Yangtze River Bridge and so on. But in the construction, there are some problems shown as follow:

- For the bi-purposed cable-stayed bridge with large-span structure, there are masses of key points in the process of construction, which involves high technology.

- Large amount of data makes it difficult to store, transfer, and exchange the information.

- Complex operation leads to the difficulty of coordination and communication.

- The construction period is long and process contain uncontrollability. So the management of process and resource is fragmentary.

- Multifarious structural style and high accuracy process and making results in significant risk and complicated technology.

So, it is imperative to establish a monitoring cloud platform used in the bridge construction based on BIM, internet of things, cloud platform, mobile terminal, and other modern information technology and lean management idea. This cloud platform can be used to capture the construction information accurately in real time, store large amount of data centrally, simulate the complicated construction procedure, and analyze data in order to early warn, monitor the risk source, control the redlining of key panel points, and reduce the danger and quality issues. And then the cooperation efficiency of each cooperated-building parties is improved significantly, and also the control of construction progress, engineering quality, safety and risk.

\section{Principle}

Based on the simulation and direction before construction, the BIM which is used in construction stage is investigated less during the construction.

The core of this cloud platform is BIM technique, the architecture is cloud platform, the basis is sensing technology, transmission mode is mobile internet, and the monitoring object is bridge construction. This cloud management platform based on BIM could track automatically the bridge construction information in real-time, analyze the data professionally, administrate the integrated 
informations, and assess and warn about the security risk, so that the construction management of bridge is transformed into 'dynamic application containing prejudgments before construction and supervision in construction'.

\section{Integration of bridge design and construction}

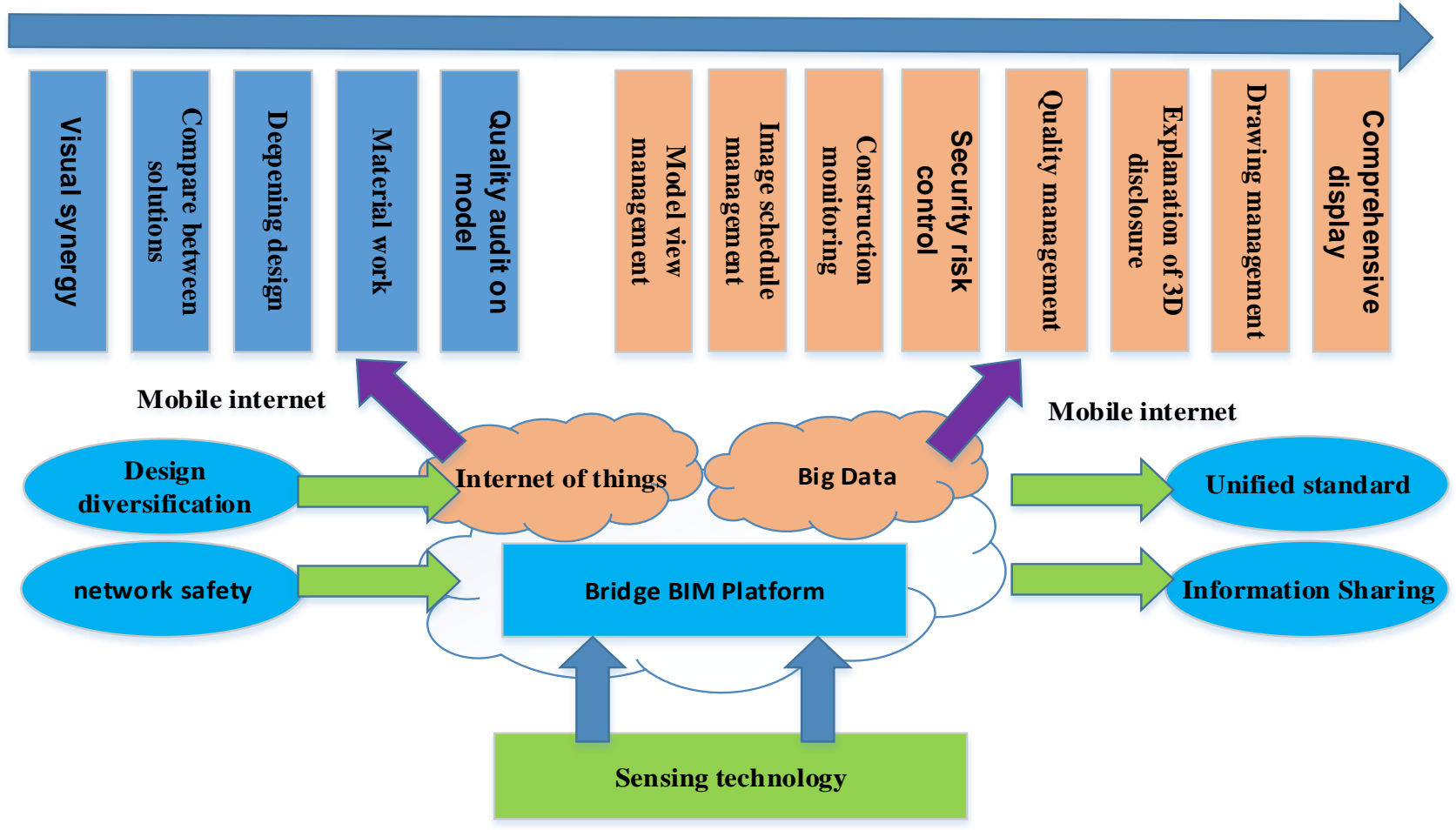

Fig.1. Principle of the system implementation

\section{Key technology}

\section{Application standard of bridge BIM}

The BIM modeling standard, delivery standard, and application standard of bridge construction are set according to "Standard for EBS and IFD of Railway Engineering Information Model" and "Guidance on Railway Engineering Breakdown Structure-EBS", which are enacted by China Railway BIM Alliance.

The precision and granularity of bridge modeling are governed by construction scheme, for instance, the member of pier body that needs multiple placing should be modeled sectionally. Before imported into the cloud platform, the model properties should be complete, such as EBS, quantities, material type, code, site, mileage, drawing number, and so on. Then construction simulation, schedule simulation, and progress tracking management could be conducted in order to ensure the accuracy and of statistical quantities. And then, the visualizations of bridge could be improved through track and accessory structure modeling.

The bridge main body model, bridge element model, Rebar model and Monitoring model are established using Tekla. The model shown in Fig.2.

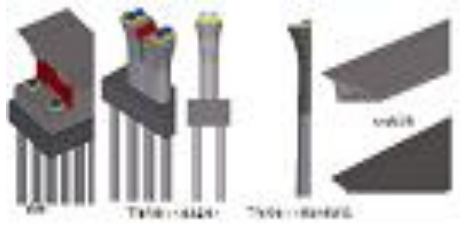

Bridge element model

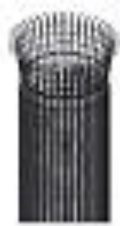

Rebar model

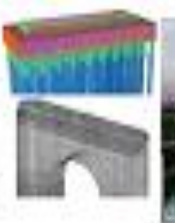

Fig.2. Bridge modeling

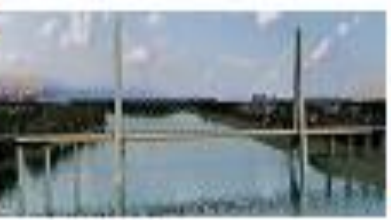

Bridge main body model

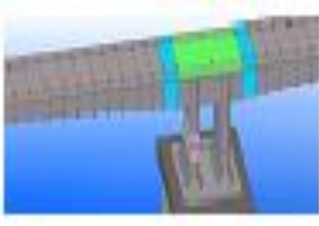

Monitoring model 


\section{Collision check and drawing optimization}

Position conflict should be checked through visual inspection and collision checking, as shown in Fig.3. The position conflict contains three types:

- Position conflict could not be adjusted during construction, such as position between steel Bridge element, and the position between steel element, steel bar and embedded parts;

- Position conflict could be adjusted during construction, such as collision between steel bar;

- There is no position conflict but the construction space is not adequate.

Collision problem could be discovered ahead of time using collision checking, and could be solved using design alteration and drawing optimization, so the risk caused by design defect could be eliminate. Also, rework can be prevented and the time and vigor consume less in order to reduce the cost.

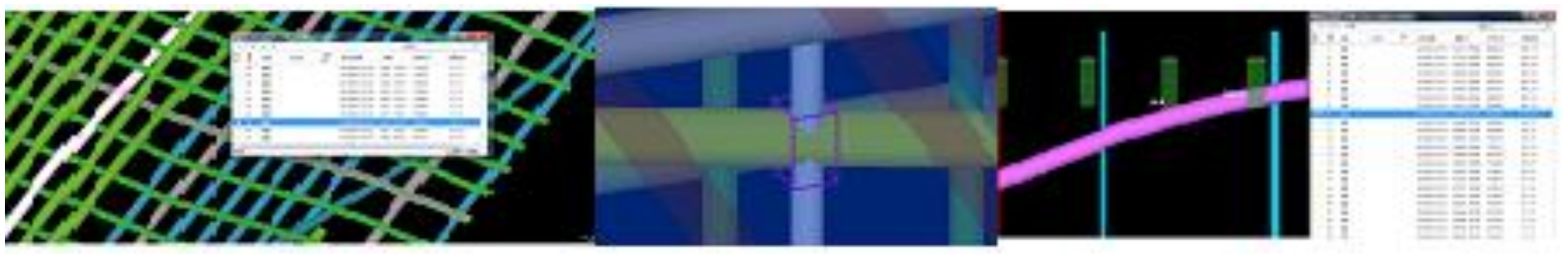

Fig.3. Bridge model collision check

\section{Lightweight processing}

As the key of the bridge BIM model, the lightweight processing must decrease the model file to one tenth of source files, ensuring the transmission and application efficiently under the premise that properties and geometry data contained in the bridge model do not lose. The flowsheet is given in Fig.4.

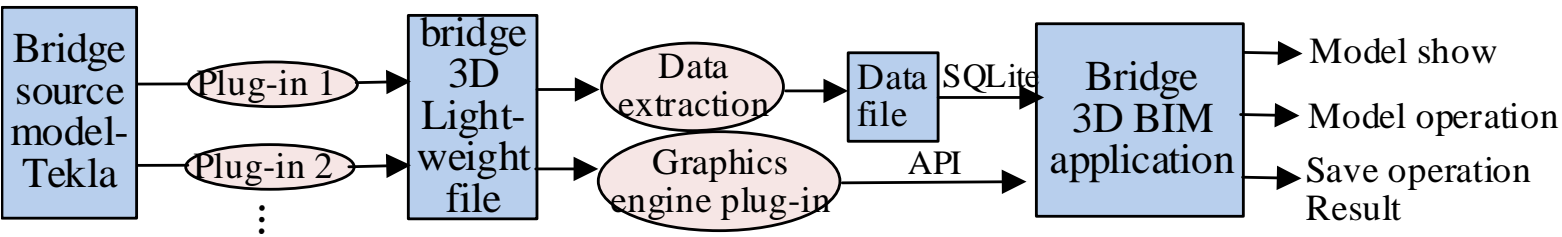

Fig.4. Lightweight treated realization process

By means of 3D lightweight processing plug-in, BIM model could be simplified. There are three purposes as shown below:

- Under the premise that the data is complete, the source file is simplified and compressed.

- Data extraction and storage: all the attribute data, attribute type information, relation between submodels, bridge elements and parts could be extracted and stored in a database intensely.

- Exhibition and operation: viewpoint switching, scaling, profiling, border blanking, hyalinizing, labeling and measuring.
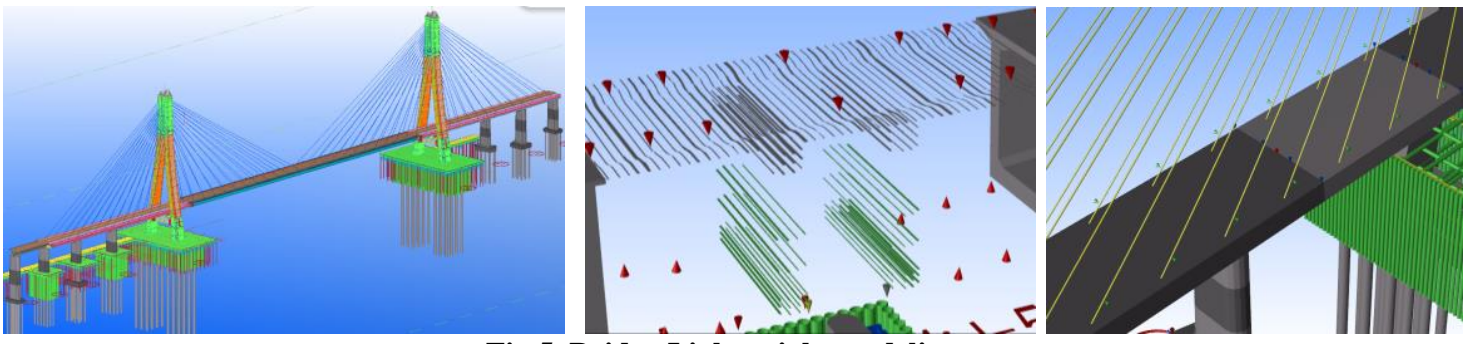

Fig.5. Bridge Lightweight modeling

\section{Management based on BIM}

To feed the demand, the management mode and application mind based on BIM should be established. The model management is shown in Fig.6. 
- The model navigation should be finished through adding the model of different bridge elements and the whole bridge.

- The bridge model elements should match corresponding 2D drawing, in order to view the 2D drawing corresponding to the different bridge elements conveniently.

- The properties of bridge model elements should be check, such as geometrical information, material property, EBS and so on.

- Custom current view could be saved for checking next time conveniently.

\begin{tabular}{|c|c|c|c|}
\hline $\begin{array}{c}\text { The bridge } \\
\text { Model navigation }\end{array}$ & $\begin{array}{c}\text { The relevant } \\
\text { drawings }\end{array}$ & $\begin{array}{l}\text { The bridge } \\
\text { element attributes }\end{array}$ & view \\
\hline $\begin{array}{l}\text { Complete model } \\
\text { which can be } \\
\text { added worksite }\end{array}$ & $\begin{array}{l}\text { Check the drawing } \\
\text { corresponding to } \\
\text { worksite }\end{array}$ & $\begin{array}{l}\text { Check bridge } \\
\text { element attributes, } \\
\text { volume, EBS code }\end{array}$ & $\begin{array}{l}\text { Saves the current } \\
\text { view for check } \\
\text { next time }\end{array}$ \\
\hline
\end{tabular}

Fig.6. the bridge Modeling management

\section{Visual schedule management based on network diagram}

According to the calculation principle of schedule network diagram, the 3D visual schedule management with an EBS core coalesces bar charts, network diagram, 3D BIM model and properties, as Fig.7 shown.

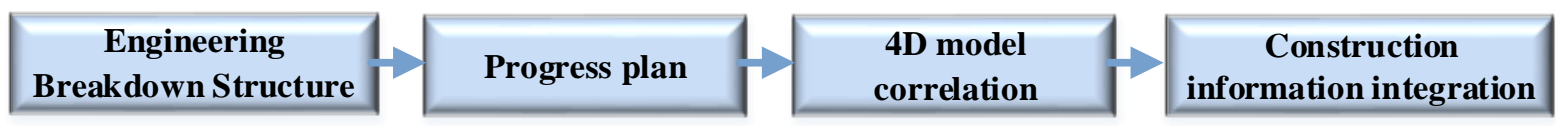

Fig.7. visualization of bridge construction progress control

The contrastive analysis between actual progress and target advance in a specific time period could be conducted by comparing the curves with different colors. The BIM model could be used to investigate the reason why the progress of workers, machines and material is different. The critical path optimization and schedule variance checking could be conducted through network diagram, in order to strengthen the control of project schedule. What's more, combining the electronic construction log, this platform can check item project working capacity, and could obtain the construction figure progress of the overall bridge project further.

\section{Health monitoring based on internet of thing technology}

App of mobile devices and server could connected to monitoring equipment, such as total station and level via integrated interface, and then the settlement could be collected in real time. Also, the real time stress can be tracked through the electronic construction log, and the data, which is actual, accurate and timely, is transmitted to cloud platform via internet automatically, as Fig.8 shown. The $3 \mathrm{D}$ visualization, real time dynamic exhibition, and transfinite pre-alarm of health monitoring avail engineers to discover and analyze the construction security in order to reduce the engineering risk.
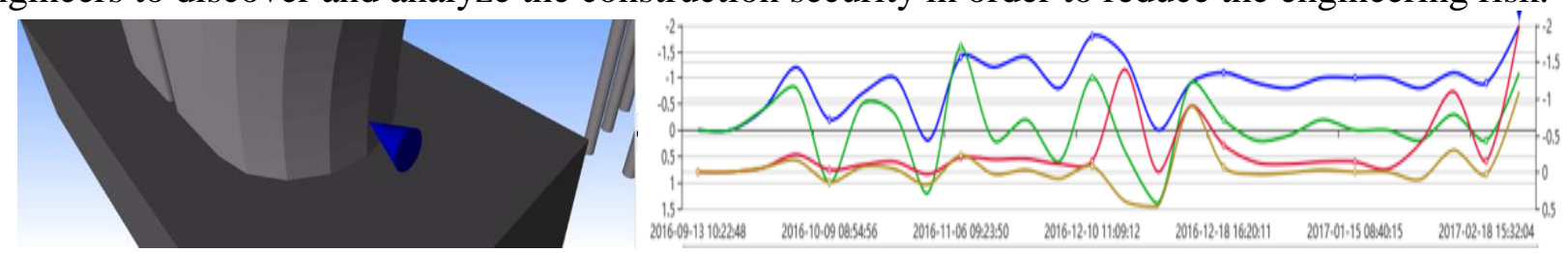

Fig.8. Bridge health monitoring based on Internet of things

\section{App of mobile devices}

App of mobile devices contributes to discuss bridge BIM model and animation simulation aided technical disclosure, and to ensure quality. The BIM model is imported into the App of mobile devices, so the App can help to layout fieldwork and compare with the entity in order to find out the quality problem intuitively. The quality defect could be recorded through taking pictures, and then all the problems are aggregated to generate rectification notice used to issue. So the engineers deal with 
problems in real-time, and strengthen the control of quality during construction. The construction templates, key points of quality control, simulation animation of construction, and the site layout are shown with BIM, in order to serve field quality control.

\section{Design and application}

The cloud platform is designed and developed with a series of advanced mainstream technology, for instance, loose-coupling SOA framework, .net-development platform, XML-data interaction, and FLEX, so that it could be applied to various cases and service object.

The primary function contains BIM view management, image progress management, construction monitoring, security risk control, quality management, drawings Management, 3D technology explanation, comprehensive display, and so on, as Fig.9 shown.

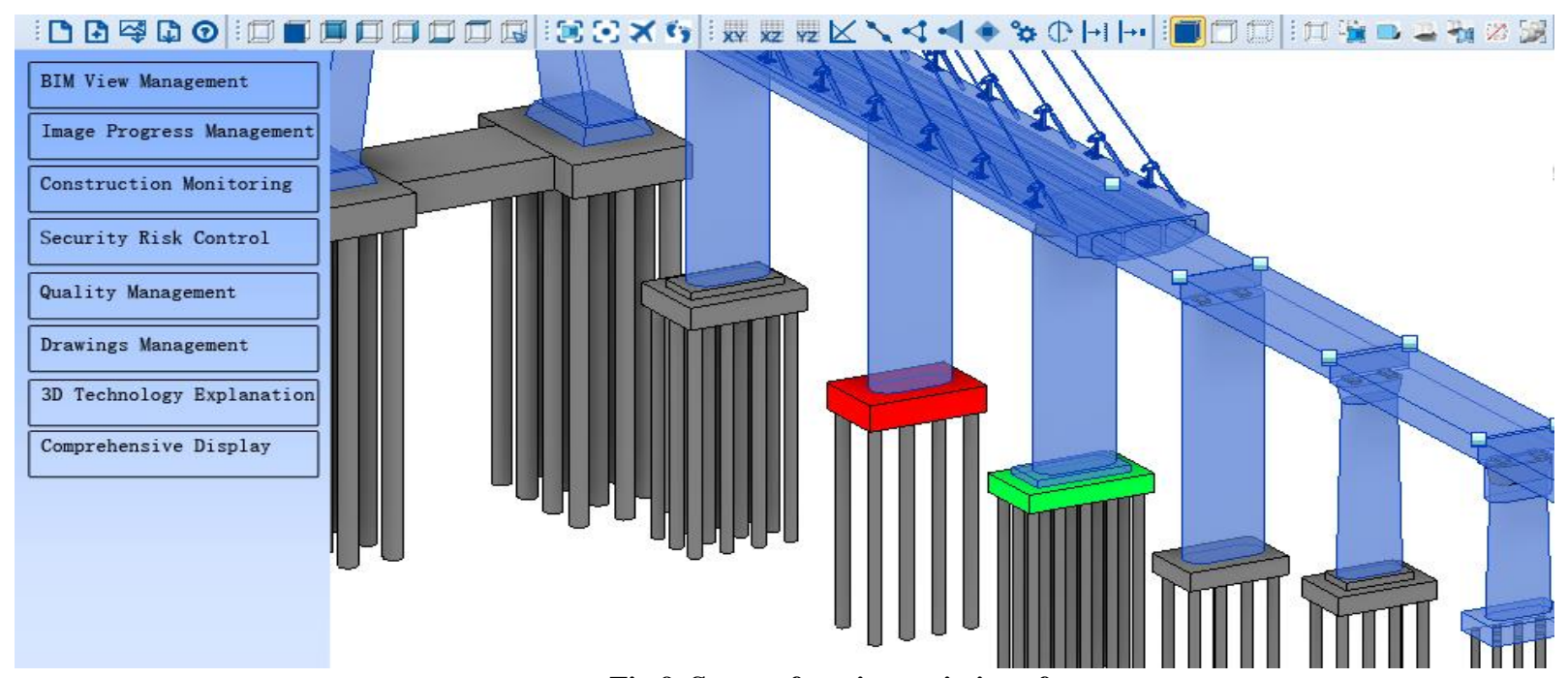

Fig.9. System function main interface

In practical application, this platform based on BIM, could capture accurate engineering information in real-time. What's more, it also could store the data and share data to improve efficiency of all the participants, promote the engineering progress, and reduce the security risk, as Fig.10 shown. The effects can be concluded as follow:

- The EBS code could be integrated with BIM model, so that establish a corresponding relationship between BIM model and EBS code. And the operative criteria of long-span bridge BIM model.

- 160 collision points of bridge prestressed steel, which affect the bridge quality, are discovered in advance through collision check, an then the design alteration is conducted ahead of time, reducing the construction rework, saving construction period, and ensuring the construction quality.

- Based on graphics lightweight technology, the platform can compress the model to 1:50. So it lower the threshold of model application.

- The BIM platform integrates the bridge health monitoring information, such as stress of the bridge cable, steel cofferdam monitoring, temperature of mass concrete, settlement of pile foundation. 178 safety risk precautions have conducted since 3 month ago.

- The 3D integrated management platform based on BIM, explicates the key construction method, controls the security risk in real-time, masters the project ' $\mathrm{s}$ image progress, and manage critically.

- The management sharing platform, based on BIM improves daily collaborative management efficiency of all the participants, decreases time for communication and daily routine check. 


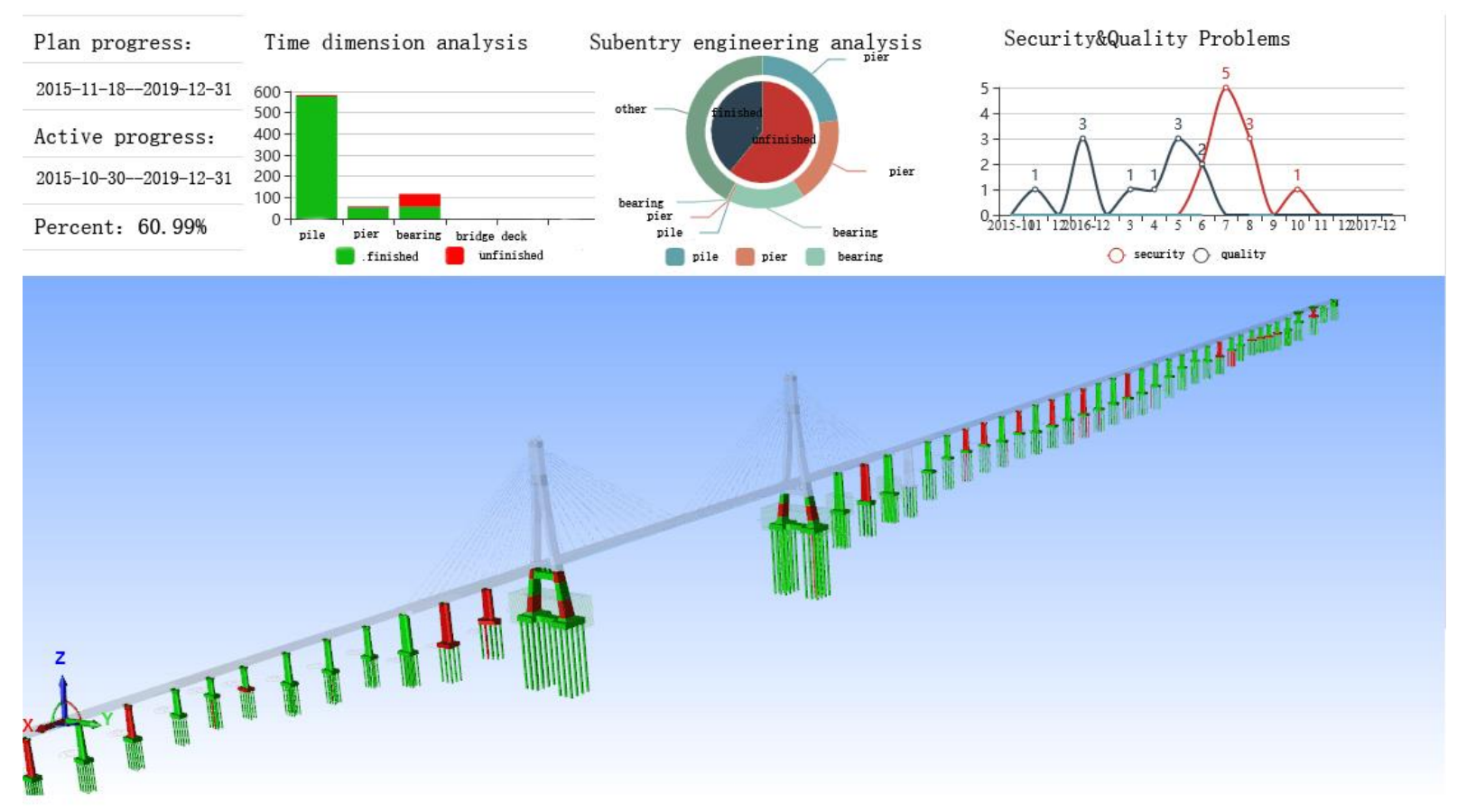

Fig.10. comprehensive information show

\section{Conclusions}

Integrating BIM, mobile internet, cloud platform, mobile terminal and other new technology, the regulatory cloud platform for bridge construction, could capture the accurate engineering information in real-time in order to control the construction process reasonably. Based on the 3D show of bridge construction quality, security, risk, and schedule, the engineering dynamic status could be controlled in real-time. The APP could assign missions in order, and find the problems in time. The platform also can eliminate information isolated island, in order to make all participants share information, benefit together. The BIM application improves to dynamic from static state. The engineering information management level of bridge construction and subtle decision-making ability could be improved. And realize standardization, informatization, and lean of project management gradually.

\section{Acknowledgements}

This work was financially supported by the China railway corporation research and development of science and technology plan project "The key technologies studies of the railway engineering construction and management based on BIM" (2016X002).

\section{References}

[1] China Railway BIM Alliance: Standard for EBS and IFD of Railway Engineering Information Model Version 1.0 (Railway Technical Innovation, Beijing 2014).

[2] China Railway BIM Alliance: Guidance on Railway Engineering Breakdown Structure-EBS (Railway Technical Innovation, Beijing 2014).

[3] L. Zhansheng, Z. Xuefeng: BIM Technology and construction project management (China Electric Power Press, Beijing 2015). 